\title{
Meta-analysis methods and models with applications in evaluation of cholesterol-lowering drugs
}

\author{
Ming-Hui Chen ${ }^{a}$, Joseph G. Ibrahim ${ }^{b,},{ }^{*} \dagger$, Arvind K. Shah ${ }^{c}$, Jianxin Linc, and Hui Yao ${ }^{\mathrm{a}}$ \\ aDepartment of Statistics, University of Connecticut, Storrs, CT, U.S.A \\ bDepartment of Biostatistics, University of North Carolina, Chapel Hill, NC, U.S.A \\ cClinical Biostatistics, Merck Research Laboratories, Rahway, NJ, U.S.A
}

\section{Abstract}

\begin{abstract}
In this paper, we propose a class of multivariate random effects models allowing for the inclusion of study-level covariates to carry out meta-analyses. As existing algorithms for computing maximum likelihood estimates often converge poorly or may not converge at all when the random effects are multi-dimensional, we develop an efficient expectation-maximization algorithm for fitting multi-dimensional random effects regression models. In addition, we also develop a new methodology for carrying out variable selection with study-level covariates. We examine the performance of the proposed methodology via a simulation study. We apply the proposed methodology to analyze metadata from 26 studies involving statins as a monotherapy and in combination with ezetimibe. In particular, we compare the low-density lipoprotein cholesterollowering efficacy of monotherapy and combination therapy on two patient populations (naive and non-naïve patients to statin monotherapy at baseline), controlling for aggregate covariates. The proposed methodology is quite general and can be applied in any meta-analysis setting for a wide range of scientific applications and therefore offers new analytic methods of clinical importance.
\end{abstract}

\section{Keywords}

aggregate covariates; heterogeneity; normal meta-regression models; multiple trials; random effects; variable selection

\section{Introduction}

An estimated 12 million Americans are taking cholesterol-lowering drugs, and experts are recommending that another 23 million should be taking them. The National Cholesterol Education Program has issued treatment guidelines identifying low-density lipoprotein cholesterol (LDL-C, 'bad' cholesterol) as a causative factor for coronary heart disease and as the main target for cholesterol-lowering and lipid-lowering therapy. Cholesterol-lowering medicines called 'statins' work mainly in the liver to decrease the production of cholesterol and reduce cholesterol in the bloodstream. This class of drugs includes: atorvastatin (Lipitor), simvastatin (Zocor), lovastatin (Mevacor), rosuvastatin (Crestor), pravastatin (Pravachol), and fluvastatin (Lescol). On the other hand, a drug called ezetimibe (Zetia) works in the digestive tract. It is unique in the way it helps block absorption of cholesterol

Copyright (C) 2012 John Wiley \& Sons, Ltd.

${ }^{*}$ Correspondence to: Joseph G. Ibrahim, Department of Biostatistics, University of North Carolina, McGavran-Greenberg Hall, Chapel Hill, NC 27599, U.S.A.

†ibrahim@bios.unc.edu 
that comes from food. Ezetimibe (EZE) can complement statins in targeting both sources of cholesterol.

In general, statins positively affect the lipid profile by increasing high-density lipoprotein cholesterol (HDL-C, 'good' cholesterol) and decreasing LDL-C and triglycerides (TG). Clinical studies have shown that statins significantly reduce the risk of heart attack and death in patients with coronary artery disease and can also reduce cardiac events in patients with high cholesterol levels. Statins are the first-line treatment in most patients, but lipid goals are frequently not achieved because of inadequate response to therapy, poor compliance, or concerns regarding the increased potential for side effects at higher doses. EZE can be given as monotherapy to lower cholesterol levels in patients who are intolerant to statin or in whom treatment with statin is not appropriate. EZE can also be used in combination with a statin in patients whose cholesterol levels remain elevated despite treatment with statin alone. It can be either co-administered with the statin dose or given as a fixed-dose combination tablet (known as Vytorin) containing EZE and simvastatin.

There has been a rich literature on statistical methods using random effects models for metaanalysis for the past 20 years. Now, random effects modeling for meta-analysis has become well accepted (e.g., [1-12]). A number of meta-analyses investigating efficacy and safety of various statins have been reported in the literature over the last 5 years. See, for example, [13, 14], the Cholesterol Treatment Trialists Collaboration Group [15], and [16]. There have been also meta-analyses reported in the literature comparing safety and efficacy of monotherapy with statins versus combination therapy of EZE added to statins (coadministration or combination tablet). Mikhailidis et al. [17] performed a meta-analysis to investigate the cholesterol-lowering effect of EZE added to ongoing statin therapy. Another meta-analysis involving 14 clinical trials was done by Catapano et al. [18] to investigate the lipid-altering efficacy of EZE co-administered with simvastatin compared with Rosuvastatin.

Common features of the meta-analyses cited previously are a lack of incorporation of (or adjusting for) any covariates through modeling and the lack of accommodating and assessing several types of treatments across studies. Also, most of the meta-analyses conducted in the literature are for adverse event comparisons, and such analyses use outcome measures of either odds ratios or relative risks. An outcome measure of mean difference is frequently used for efficacy comparisons, which is our primary analysis and outcome measure in this paper. While carrying out a meta-analysis, it is crucial to account for and understand differences among the studies to be combined with respect to study design, duration of treatment, durations of follow-up, and patient population, which is a function of entry criteria. As can be seen from Tables I-III, the treatment arms, treatment durations, and patient entry criteria for the studies considered here varied considerably, warranting inclusion of covariates into the model. The study-level aggregate estimates available from individual publications (listed in Tables I and II) and used in the metaanalysis here were indeed adjusted for selected covariates, but the set of selected covariates varied from study to study. Thus, the available aggregate estimates were not uniformly adjusted and again warrant the inclusion of important covariates in the meta-analysis.

There are some recent developments on meta-regression models. See, for example, [19-26]. Compared with the current literature on meta-regression, our contributions in this paper are several-fold. First, we develop novel meta-analysis models on the basis of multi-dimensional random effects that allow for study-level covariates and general covariance matrices for the random effects. Next, we develop an efficient expectation-maximization (EM) algorithm for fitting these models and develop methods for examining heterogeneity and variable selection for these models. Specifically, for assessing heterogeneity, we generalize Cochran's $Q$ 
statistic [27] to meta-analysis regression models with covariates and multi-dimensional random effects in order to test homogeneity among studies. Finally, we have assembled a dataset on the basis of 26 large clinical studies and have applied the proposed methods to this dataset in order to address some important clinical questions. We organize the rest of this paper as follows. In Section 2, we give a detailed description of the LDL-C data on the basis of the 26 studies and discuss the specific hypotheses of interest for these studies. In Section 3, we give the full development of the meta-analysis regression models with multidimensional random effects in Section 3.1, present an extension of Cochran's $Q$ in Section 3.2 , and provide the necessary mathematical formulation of three variable selection criteria in Section 3.3, respectively. In Section 4, we carry out an extensive simulation study to examine the performance of the proposed methods. Section 5 presents a detailed analysis of the LDL-C data, and we conclude the paper with some discussion and extensions of the proposed methodology in Section 6. The detailed development of the EM algorithm including closed-form expressions for the E-step and the estimates in the M-step is given in the Appendix.

\section{Low-density lipoprotein cholesterol data}

In this paper, we carry out aggregate data meta-analyses to evaluate the LDL-C lowering effect of EZE in combination with statin in comparison with statin alone in randomized controlled trials on treatment-naïve patients at baseline (on a first-line therapy) and those continuing on statins at baseline (on a second-line therapy). The objective is to evaluate the LDL-C lowering effect of EZE added to statin versus statin in treatment-naïve patients or EZE added to baseline statin versus placebo added to baseline statin in statin-treated patients. The study inclusion criteria were Merck-sponsored double-blind, randomized, active or placebo-controlled clinical trials on adult patients with primary

hypercholesterolemia.

These criteria led to 26 studies whose titles, treatment groups, treatment durations, number of patients, and the citations of primary publications are given in Table I for the first-line therapy studies and Table II for the second-line therapy studies. These trials were conducted between November 1999 and October 2008, and study durations ranged from 4 to 24 weeks. Some trials had longer durations with titration of doses, but only the data prior to the first titration were used in the analyses. The primary end point in these trials was the percent reduction in LDL-C from baseline. The entry criteria for the patients in each of these studies are summarized in Table III. Figure 1 shows the forest plots of the metadata for these 26 studies. Note that the reported means were model-based means. In practice, the patient-level data are not available to the researchers, and hence our focus here is on aggregate data metaanalysis instead of patient-level data meta-analysis.

For the analyses, different statins and their doses are combined to form the statin and (statin + EZE) treatment groups. EZE is available at only one dose of $10 \mathrm{mg}$, and the statins used in these studies included simvastatin, atorvastatin, lovastatin, rosuvastatin, pravastatin, and fluvastatin. The following hypotheses are of interest: $H_{01}$ : No treatment differences (addition of EZE has no effect) with respect to LDL-C lowering (percent reduction) in the first-line therapy studies; $H_{02}$ : No treatment differences (addition of EZE has no effect) with respect to LDL-C lowering (percent reduction) in the second-line therapy studies; and $H_{03}$ : Treatment differences within the first-line and second-line therapy studies are the same. 


\section{Methods for meta-analysis with multi-dimensional random effects and aggregate covariates}

\subsection{The meta-analysis regression model}

Consider $K$ randomized trials (studies), where each trial has $J$ treatment arms, and patients in each trial are either all on the first-line therapy or the second-line therapy prior to the trial. Let $y_{j k}$ denote the aggregate response with a known precision parameter $w_{j k}$. Let $I_{k}$ be the indicator function such that $I_{k}=1$ if patients are on the second-line therapy and $I_{k}=0$ if patients are on the first-line therapy. Also let $\boldsymbol{x}_{j k}$ denote a $p$-dimensional vector of aggregate covariates for $j=1,2, \ldots, J$ and $k=1,2, \ldots, K$. Then, we propose the following normal random effects model for the meta-analysis:

$$
y_{j k}=\boldsymbol{x}_{j k}^{\prime} \beta+\left(1-I_{k}\right) \gamma_{1 j k}+I_{k} \gamma_{2 j k}+\varepsilon_{j k},
$$

where $\beta=\left(\beta_{1}, \beta_{2}, \ldots, \beta_{p}\right)^{\prime}$ is the vector of fixed effects regression coefficients corresponding to the $p$ aggregate covariates and

$$
\varepsilon_{j k} \sim N\left(0, w_{j k}^{-1}\right)
$$

independently, for $j=1,2, \ldots, J$ and $k=1,2, \ldots, K$. Let $\gamma_{R, 1 k}=\left(\gamma_{1 k 1}, \gamma_{1 k 2}, \ldots, \gamma_{1 k J}\right)^{\prime}$, and $V_{R, 2 k}=\left(V_{2 k 1}, V_{2 k 2}, \ldots, V_{2 k J}\right)^{\prime}$, which represent the vectors of random effects of the first-line and second-line therapies for the model. Thus, $\gamma_{R, k}=\left(\gamma_{R, 1 k}^{\prime}, \gamma_{R, 2 k}^{\prime}\right)^{\prime}$ represents the vector of random effects for the model. We assume $\gamma_{R, 1 k}$ and $Y_{R, 2 k}$ are independent, and

$$
\gamma_{R, \ell k}=\gamma_{\ell}+\xi_{\ell k}, \quad \xi_{\ell k}=\left(\xi_{\ell 1 k}, \ldots, \xi_{\ell J k}\right)^{\prime} \sim N_{J}\left(\mathbf{0}, V_{\ell}\right)
$$

where $V_{\ell}=\left(V_{\ell j j}\right)$ is an unknown $J \times J$ covariance matrix and $\gamma_{\ell}=\left(\gamma_{\ell 1}, \ldots, \gamma_{\ell J}\right)^{\prime}$ denotes the vector of the overall treatment effects when patients were on the $\ell$ th line therapy for $\ell=1,2$. Let $\xi_{k}=\left(\boldsymbol{\xi}_{1 k}^{\prime}, \boldsymbol{\xi}_{2 k}^{\prime}\right)^{\prime}$ and $\gamma=\left(\gamma_{1}^{\prime}, \gamma_{2}^{\prime}\right)^{\prime}$. Using (3.3), we have $\boldsymbol{\gamma}_{R, k}=\boldsymbol{\gamma}+\boldsymbol{\xi}_{k}$ and $\boldsymbol{\xi}_{k} \sim N_{2 \boldsymbol{\lambda}}(\mathbf{0}, V)$, where $V=\operatorname{diag}\left(V_{1}, V_{2}\right)$.

We have proposed here a very general class of meta-regression models that are suited for analyzing metadata from studies involving first-line and second-line therapies. Such studies are becoming quite prominent in diabetes, cardiovascular, and cancer clinical trials where patients received a prior therapy that is identical or nearly identical to the therapy for the current clinical trial. Such types of studies pose many new challenges in the meta-regression setting such as multivariate and correlated random effects that lead to potentially high dimensional variance components. The meta-analysis regression model defined by (3.1), (3.2), and (3.3) is very general, which can accommodate several treatments in first-line and second-line as well as a large number of covariates. However, the resulting model requires estimation of the covariance matrix of the random effects, denoted by $V$, which is a block diagonal matrix. Simultaneous estimation of $(V, \gamma, \beta)$ is not trivial and requires a sophisticated and computationally intensive EM algorithm, which is developed in the Appendix.

\subsection{Test of statistical homogeneity}

The hypotheses for heterogeneity across $K$ studies are $H_{0}: V=0$ versus $H_{1}: V>0$, where $V$ $>0$ denotes that $V$ is a positive definite matrix. In this regard, we extend the $Q$ test statistic 
for homogeneity proposed by Cochran [27] to meta-analysis regression models with multivariate random effects. In (3.3), when $V=0, \gamma_{R, 1}=\cdots=\gamma_{R, K}=\gamma$, indicating that there is no heterogeneity across the $K$ studies. As $V_{R, k}$ in (3.3) is a $q$-dimensional vector of random effects, the classical $Q$ function of Cochran [27] is not well defined. In this case, we propose the following extension of the $Q$ test statistic:

$$
Q_{K}=\sum_{k=1}^{K}\left(\boldsymbol{y}_{k}-X_{k}^{*} \hat{\theta}\right)^{\prime} W_{k}\left(\boldsymbol{y}_{k}-X_{k}^{*} \hat{\theta}\right)
$$

where $\hat{\theta}=\left(\sum_{k=1}^{K} X_{k}^{*^{\prime}} W_{k} X_{k}^{*}\right)^{-1} \sum_{k=1}^{K} X_{k}^{*^{\prime}} W_{k} \boldsymbol{y}_{k}, \boldsymbol{y}_{k}=\left(y_{1 k}, \ldots, y_{J k}\right)^{\prime}, W_{k}=\operatorname{diag}\left(w_{1 k}, \ldots\right.$, $\left.W_{J k}\right), X_{k}^{*}=\left(X_{k}, Z_{k}\right)$ is a $J \times(p+2 J)$ matrix, $X_{k}=\left(\boldsymbol{x}_{1 k}, \ldots, \boldsymbol{x}_{J k}\right)^{\prime}$ is a $J \times p$ matrix, $\boldsymbol{Z}_{k}=\left(z_{1 k}, \ldots\right.$, $\left.z_{J k}\right)^{\prime}$ is the $J \times 2 J$ matrix, and $z_{j k}=\left(\left(1-I_{k}\right) \boldsymbol{e}_{j}^{\prime}, I_{k} \boldsymbol{e}_{j}^{\prime}\right)^{\prime}$, where $\boldsymbol{e}_{j}=(0, \ldots, 1, \ldots, 0)^{\prime}$ with a 1 in its $j$ th component and 0 elsewhere, for $j=1, \ldots, J$. Similar to [27], we can show that under the hypothesis of homogeneity, $V=0$, and assuming that the regression model is correct, $Q_{K} \sim \chi_{J K-2 J-p}^{2}$. Thus, if the regression model is correct, a rejection of the null hypothesis implies that there is heterogeneity across the $K$ studies. However, without knowledge of whether the regression model is correct, a rejection of the null hypothesis implies either heterogeneity or misspecification of the regression model.

\subsection{Variable selection for meta-analysis regression models}

Let $\eta=\left(\varphi, V^{-1}\right)$, where $\varphi=\left(\boldsymbol{\beta}, \boldsymbol{V}^{\prime}\right)^{\prime}$, denotes the collection of all the unknown parameters.

Write $\tilde{\boldsymbol{x}}_{j k}=\left(\boldsymbol{x}_{j k}^{\prime}, \boldsymbol{z}_{j k}^{\prime}\right)^{\prime}$ for $j=1, \ldots, J$ and $X_{k}=\left(\boldsymbol{x}_{\rrbracket_{k}}, \boldsymbol{x} \mathbb{Q}_{k}\right)^{\prime}$. For the meta-regression model defined by (3.1), (3.2), and (3.3), the marginal distribution of $\boldsymbol{y}_{k}$ is analytically available.

After some algebra, we obtain

$f\left(\boldsymbol{y}_{k} \mid X_{k}, Z_{k}, \phi, V\right)=\frac{\left|W_{k}^{-1}+Z_{k} V Z_{k}^{\prime}\right|^{-\frac{1}{2}}}{(2 \pi)^{J / 2}} \exp \left\{-\frac{1}{2}\left(\boldsymbol{y}_{k}-X_{k} \phi\right)^{\prime}\left(W_{k}^{-1}+Z_{k} V Z_{k}^{\prime}\right)^{-1}\left(\boldsymbol{y}_{k}-X_{k} \phi\right)\right\}$.

Thus, the observed-data log-likelihood is given by

$\ell_{\mathrm{obs}}(\eta)=-\frac{J K}{2} \log (2 \pi)-\frac{1}{2} \sum_{k=1}^{K}\left\{\log \left|W_{k}^{-1}+Z_{k} V Z_{k}^{\prime}\right|+\left(\boldsymbol{y}_{k}-X_{k} \phi\right)^{\prime}\left(W_{k}^{-1}+Z_{k} V Z_{k}^{\prime}\right)^{-1}\left(\boldsymbol{y}_{k}-X_{k} \boldsymbol{\phi}\right)\right\}$.

We consider three variable selection criteria for selection of the covariates in our metaregression model, namely, the Akaike information criterion (AIC), Bayesian information criterion (BIC), and a finite-sample corrected version of AIC (AICC). These criteria are computed as follows:

$\mathrm{AIC}=-2 \ell_{\mathrm{obs}}(\widehat{\eta})+2 q, \mathrm{AICC}=-2 \ell_{\mathrm{obs}}(\widehat{\eta})+2 q(J K) /(J K-q-1), \mathrm{BIC}=-2 \ell_{\mathrm{obs}}(\widehat{\eta})+q \log (K)$,

where $\eta$ is the MLE of $\eta$ and $q=p+2 J+J(J+1)$ is the dimension of $\eta$. See [28] and [29] for additional details. We mention here that in our variable selection procedure, $\boldsymbol{V}$ and $V$ are always kept in every model and, therefore, are never candidates for selection. Our variable 
selection procedure is only concerned with selecting the covariates $\boldsymbol{x}_{j k}$, that is, components of $\beta$

\section{A simulation study}

To examine the performance of the proposed method, we carry out a simulation study with $K=26$ trials and four trial-level covariates. Among the 26 trials, 13 are the first-line therapy trials, and the remaining 13 are the second-line trials. Within each trial, two treatment arms $(J=2)$ are assumed. The sample sizes, $n_{j k}$, were chosen to be the same as those in the LDL$\mathrm{C}$ data discussed in Section 2. The within-subject standard deviations were set to the values of the standard errors (SEs), $\mathrm{SD}_{j k}$, estimated from the LDL-C data. We used the values of baseline LDL-C, baseline HDL-C, age in years, and duration in weeks from the LDL-C data for these four covariates. Then, we generated the simulated data from the nine metaregression models defined by (3.1), (3.2), and (3.3) with various combinations of $\beta$ and $V$. Specifically, $\gamma_{11}=-52, \gamma_{12}-\gamma_{11}=-12, \gamma_{21}=-23$, and $\gamma_{22}-\gamma_{21}=-20$ were used in all the nine meta-regression models. To evaluate the performance of the model selection criteria, we considered three covariate models (C-Models), namely, C-Model I: $\boldsymbol{\beta}=(0.1,-0.7,0.6$, 1.0) (four covariates); C-Model II: $\boldsymbol{\beta}=(0.1,-0.7,0.0,1.0)$ (three covariates); and C-Model III: $\boldsymbol{\beta}=(0.1,0.0,0.0,1.0)$ (two covariates), respectively. For each covariate model, we considered three different values of $V$ (the covariance matrix of the random effects) such that $V=V_{0}$ (large between-study variability), $V=V_{0} / 2$ (moderate between-study variability), and $V=V_{0} / 10$ (small between-study variability), where $V_{0}=\operatorname{diag}\left(V_{01}, V_{02}\right)$, $V_{01}=\left(\begin{array}{cc}36 & -12 \\ -12 & 11\end{array}\right)$ and $V_{02}=\left(\begin{array}{cc}18 & -16 \\ -16 & 22\end{array}\right)$. For each meta-regression model, we generated 1000 simulated data sets, and for each simulated dataset, we fit 16 models. AIC, AICC, and BIC were computed for all 16 models, whereas the estimates of the model parameters were computed, and the test of statistical homogeneity was performed only under the true model, which was used to generate the simulated data.

Let $\varphi_{j}$ denote the $j$ th component of $\varphi$. Also, let $\varphi_{1 j}^{l}$ and $\operatorname{SE}\left(\varphi_{i j}^{l}\right)$ be the maximum likelihood estimate of $\varphi_{j}$ and the SE of $\varphi_{1 j}^{\mathbb{D}}$ from the $i$ th simulated dataset for $i=1,2, \ldots, 1000$. We define the simulation estimate (EST) and the SE to be $\frac{1}{1000} \sum_{i=1}^{1000} \widehat{\phi}_{i j}$ and $\frac{1}{1000} \sum_{i=1}^{1000} \operatorname{SE}\left(\widehat{\phi}_{i j}\right)$.

We also define the root of the mean squared error (RMSE) as $\left[\frac{1}{1000} \sum_{i=1}^{1000}\left(\widehat{\phi}_{i j}-\phi_{j}^{*}\right)^{2}\right]^{1 / 2}$, where $\phi_{j}^{*}$ is the true value of $\varphi_{j}$. Table IV shows ESTs, SEs, RMSEs, and powers of $\boldsymbol{\beta}$ and $\boldsymbol{\gamma}$ under the nine combinations of $\beta$ and $V$, where the power was calculated on the basis of a significance level of 0.05 . From Table IV, we see that for all parameters, the ESTs were very close to the corresponding true values, and the SE and RMSE become smaller and the power increases when the number of covariates is less or there is less between-study variability. In particular, under C-Model III, the parameter estimates were quite accurate and had good powers given that the number of trials $(K=26)$ is relatively small. We notice that under C-Models I and II, when $V$ is large, the estimate of $\gamma_{21}$ has much lower power than that of $\gamma_{11}$, which is expected as the effect size of $\gamma_{21}$ is much smaller than the one of $\gamma_{11}$. In addition, we computed the coverage probability (CP) of the $95 \%$ confidence interval on the basis of a normal approximation. The respective CPs for $\left(\gamma_{12}^{*}=\gamma_{12}-\gamma_{11}, \gamma_{22}^{*}=\gamma_{22}-\gamma_{21}\right)$ in the 1000 simulations when $V=V_{0}, V_{0} / 2$, and $V_{0} / 10$ were $(92.3 \%, 93.4 \%),(91.6 \%, 93.5 \%)$, and $(92.4 \%, 95.0 \%)$ under C-Model I; (92.9\%, 93.2\%), (91.7\%, 93.8\%), and (92.4\%, 94.7\%) under C-Model II; and $(93.0 \%, 93.5 \%),(91.6 \%, 93.8 \%)$, and $(92.1 \%, 94.8 \%)$ under C-Model III. The CPs for the other parameters were similar and thus not reported here for brevity. 
Table $\mathrm{V}$ presents the frequencies of ranking the true model as one of the top three models under each of the AIC, AICC, and BIC criteria. From Table V, we see that AIC outperforms both AICC and BIC when the full model is the correct model and the between-study variability is large. The results in this table also indicate that the performance of all three criteria improves when the between-study variability becomes smaller, and all three criteria perform well when the between-study variability is small. Under C-Model II, the percentages of ranking the true model as one of the top three models in the 1000 simulations were $85.2 \%, 93.9 \%$, and $99.8 \%$ for AIC, $78.1 \%, 93.3 \%$, and $99.8 \%$ for AICC, and $77.5 \%$, $93.5 \%$, and $99.8 \%$ for BIC when $V=V_{0}, V_{0} / 2$, and $V_{0} / 10$, respectively. In this sense, AIC performs slightly better than AICC and BIC. When C-Model III is the correct model, both AICC and BIC perform better than AIC. When the full model is the correct model, BIC performs slightly better than AICC, whereas AICC does slightly better than BIC when CModels II and III are the correct models. Overall, AIC tends to select a larger model, whereas both AICC and BIC are in favor of smaller models.

In all nine simulation settings, we carried out the test of statistical homogeneity via the $Q$ test statistic given by (3.4). The average values of $Q_{K}$ were $1476.2,761.0$, and 188.3 for $V=$ $V_{0}, V_{0} / 2$, and $V_{0} / 10$ under C-Model I; 1633.1, 840.0, and 204.9 for $V=V_{0}, V_{0} / 2$, and $V_{0} / 10$ under C-Model II; and 1778.2, 913.0, and 220.3 for $V=V_{0}, V_{0} / 2$, and $V_{0} / 10$ under C-Model III. In all nine simulations, the $Q$ statistic rejected the hypothesis of homogeneity; that is, $V$ $=0,100 \%$ of the time in the 1000 simulated data sets, resulting in $100 \%$ power. We note

that $V_{01} / 10=\left(\begin{array}{cc}3.6 & -1.2 \\ -1.2 & 1.1\end{array}\right)$ and $V_{02} / 10=\left(\begin{array}{cc}1.8 & -1.6 \\ -1.6 & 2.2\end{array}\right)$, and, hence, the variances are relatively small compared with the effect sizes of the treatments. We also conducted a simulation study with $V=0$. That is, we generated the data from (3.1) and (3.2) with $\gamma_{1 j k}=$ $\gamma_{1 j}$ and $\gamma_{2 j k}=\gamma_{2 j}$ for $j=1,2$. Under C-Model I, the average value of $Q_{K}$ was 44.7 , and the $Q$ test rejected the hypothesis that $V=0,54$ times in the 1000 simulations at a significance level of 0.05. Note that under this simulation setting, $\chi_{J K-2 J-p}^{2}=\chi_{44}^{2}$, the 95th percentile of $\chi_{44}^{2}$ is 60.5 , and hence, the average value of $Q_{K}$ is much smaller than this chi-square percentile. This result implies that the type I error of the $Q$ test was about 5\%. For the 1000 data sets generated under C-Model I, we also fit all 15 reduced models with zero to three covariates and then performed the $Q$ test for each of these models. In this case, all 15 regression models were misspecified. The average values of $Q_{k}$ ranged from 198.3 to 635.2, the minimum values of $Q_{k}$ ranged from 120.1 to 492.7, and all $p$-values were less than 0.00001 for these 15 models. Similar results were obtained under C-Models II and III. These results further demonstrate that the $Q$ statistic can be used to test either heterogeneity or misspecification of the regression model as discussed in Section 3.2.

\section{Analysis of low-density lipoprotein cholesterol data}

For the LDL-C data, $y_{j k}$ denotes the percent reduction of LDL-C and $w_{j k}^{-1}=\mathrm{SD}_{j k}^{2} / n_{j k}$, where $\mathrm{SD}_{j k}$ and $n_{j k}$ denote the standard deviation of the mean LDL-C and the sample size, respectively, for the $j$ th treatment in the $k$ th trial. There were $K=26$ studies, the first-line and second-line therapies were 'not on statin' and 'on statin' prior to the trial, and there were two treatment arms $(J=2)$ corresponding to 'statin alone' and 'statin + EZE'. We considered eight aggregate covariates, including baseline LDL-C, baseline HDL-C, and baseline TG, age in years, white (\%), male (\%), diabetes mellitus (DM) (\%), and duration in weeks. Thus, we have $\boldsymbol{x}_{j k}=\left((\text { baseline LDL-C })_{j k} \text {, (baseline HDL-C }\right)_{j k}$, (baseline TG) ${ }_{j k}$, age ${ }_{j k}$, white $_{j k}$, male $_{j k}, \mathrm{DM}_{j k}$, Duration $\left.{ }_{j k}\right)^{\prime}$ and the vector of corresponding regression coefficients is $\beta=\left(\beta_{1}, \ldots, \beta_{8}\right)^{\prime}$ with $p=8$ under the full model. We carried out variable selection for the meta-analysis regression model given in (3.1). We computed AICs, AICCs, and BICs for all 
$2^{8}=256$ possible models. Table VI shows the values of these measures for the top three AIC models along with the no covariate model. The top three AIC models were (baseline LDL-C, baseline HDL-C, Duration), (baseline LDL-C, Duration), and (baseline LDL-C, baseline HDL-C, age, Duration), and the corresponding AIC values were 315.7, 316.6, and 316.6. The top AIC model, (baseline LDL-C, baseline HDL-C, Duration), is the third best $\mathrm{BIC}$ model with $\mathrm{BIC}=332.1$ and the sixth best $\mathrm{AICC}$ model with $\mathrm{AICC}=325.3$. The second best AIC model, (baseline LDL-C, Duration), is also the second best AICC and BIC model. The no covariate model and the full model had AIC values of 318.8 and 323.8, which were ranked 23 and 196, respectively. The no covariate model is the best AICC model as well as the best BIC model. The third best AICC model was the model with a single covariate, namely, Duration, which was the 13th best AIC model and the fourth best BIC model. The values of AICC, AIC, and BIC for the model (Duration) were 325.0, 318.5, and 332.3. As shown in the simulation study, AIC tends to select a larger model, whereas AICC and BIC are in favor of a smaller model as both AICC and BIC penalize the dimension of the model parameters more than AIC. In practice, AIC is more conservative and desirable compared with AICC and BIC in variable selection as it penalizes the dimension of the model parameters less and avoids the misidentification of potentially important covariates. Table VI also shows the values of $Q_{K}$. Thus, the $Q_{K}$ test rejects the hypothesis of no heterogeneity, which implies that there was heterogeneity across the $K=26$ studies. This was confirmed by the large values of the maximum likelihood estimates of the $V_{j j}$ s as shown in Table VII. In addition, we fit the meta-regression models with no random effects $(V=0)$, a single random effect for each of the first-line therapy trials $\left(V_{122}=0\right)$, a single random effect for each of the second-line therapy trials $\left(V_{222}=0\right)$, and a single random effect for all trials $\left(V_{122}=\right.$ $V_{222}=0$ ). Under the best AIC model, the AIC values were 1632.9, 533.4, 395.3, and 643.5 corresponding to these four types of random effects models. These AIC values were much larger than 315.7 under the model with four random effects. Similar results were obtained under other covariate models. These results further confirmed that there was heterogeneity of treatment effects within first-line and within second-line trials.

Table VII shows the parameter estimates under the best AIC model. From this table, we see that baseline LDL-C and duration were significant with $p$-values of 0.0277 and 0.0181 , respectively. The positive coefficient for baseline LDL-C in the model implies that the higher the baseline LDL-C value was, the lower the percent reduction in LDL-C. Similarly, the longer the study duration, the lower the percent reduction in LDL-C. This might be due to a full realization of the beneficial effect of statins over the first several weeks, which might be difficult to fully sustain over the long run because of a possible lack of drug compliance. The predicted percent changes from baseline in LDL-C for patients on statin and EZE/statin in first-line therapy studies were -38.50 and -50.47 , respectively. The predicted percent changes from baseline in LDL-C for patients on statin and EZE/statin in second-line therapy studies were -7.55 and -27.53 , respectively. The treatment differences for first-line and second-line therapy studies were -11.97 and -19.97 , respectively, which were statistically significant each with $p$-value $<0.0001$. The treatment difference for second-line therapy studies was significantly higher than that for first-line therapy studies. We also fitted two other models (no covariate model and a full model with all eight covariates) for sensitivity analysis. The results of these two models were consistent with the best AIC model as seen from Table VII.

Under the meta-analysis regression model specified by (3.1), (3.2), and (3.3), the three hypotheses stated in Section 2 can be mathematically written as $H_{01}: \gamma_{12}=\gamma_{11} ; H_{02}: \gamma_{22}=$ $\gamma_{21}$; and $H_{03}: \gamma_{22}-\gamma_{21}=\gamma_{12}-\gamma_{11}$. These three hypotheses were all rejected under the best AIC model as shown in Table VII. Rejection of these hypotheses implies that there were treatment differences within first-line and second-line studies, and there were unequal treatment differences between first-line and second-line studies. Rejection of these 
hypotheses also indicates that EZE had a significant effect within first-line and second-line studies and that LDL-C lowering due to statin was different between first-line and secondline studies. It is worthwhile to note that the aggregate study-level estimates used in our meta-analysis were already adjusted for baseline LDL-C (along with other covariates which varied from study to study), and yet baseline LDL-C still remained a significant covariate in both the final AIC model and the full model. This indicates differences among study populations and study characteristics and again emphasizes the importance of incorporating study-level covariates into the meta-analysis model. It should also be noted that the no covariate model is nested in the best AIC model, whereas the best AIC model is nested in the full model. The nested nature of these three models allows us to make a formal statistical comparison of the three models via likelihood ratio tests. On the basis of the likelihood ratio tests, we found that the best AIC model provided a significantly better fit than the no covariate model ( $p$-value $=0.0288$ ); however, the full model did not provide a significantly better fit than the no covariate model because of possible over-fitting of the data ( $p$-value $=$ $0.2024)$; and, in addition, the best AIC model fit the data equally well as the full model ( $p$ value $=0.8564)$. Interestingly, the maximum likelihood estimates of the treatment effects were similar in all three models.

The EM algorithm discussed in the Appendix was implemented using FORTRAN 95 with IMSL. The convergence criterion for the EM algorithm for obtaining the MLEs was that the squared distance of all the model parameters between the $t$ th and $(t+10)$ th iterations was less than $10^{-7}$. This algorithm converged in approximately 1000 iterations for all models we considered in both the simulation study and the analysis of the LDL-C data. The FORTRAN 95 code is available upon request.

\section{Discussion}

Our goal in this investigation was to develop a novel class of models for meta-analysis incorporating study-level covariates and be able to fit these models in order to study the impact of study-level covariates on the response variable. We applied this technique to an important problem in the field of cholesterol reduction and studied the effect of adding EZE to a class of drugs called statins through aggregate meta-analysis controlling for some of the important study-level covariates. Our proposed variable selection procedure led us to our best AIC model with three covariates: baseline LDL-C, baseline HDL-C, and study duration. This model provided a significantly better fit over the model with no covariates. The model implied that the higher the baseline LDL-C is, the lower the percent reduction in LDL-C at study end. Also, the longer the study duration is, the lower the LDL-C percent reduction. The latter result is somewhat of a conjecture because the duration of the studies considered here (as well as for many other randomized clinical trials on statins) did not vary over a large range. The issue of the impact of study duration remains an open question needing further investigation. It is worthwhile to reiterate the importance of incorporating covariates in the meta-analysis model even though the aggregate study-level estimates used in the meta-analysis may have already adjusted for those covariates. Differences among study populations and study characteristics necessitates the inclusion of these covariates into the analysis.

The beneficial effects of statin therapy is well studied and well established. We studied the effect of combination therapy of EZE along with statins, and our meta-analysis has allowed us to clearly demonstrate enhanced reduction in LDL-C because of addition of EZE over and above that due to statins alone. This incremental reduction is clinically as well as statistically significant. Presently, Vytorin is the only drug approved in the USA by the FDA, which combines simvastatin and EZE into one tablet. Our analysis has also established larger between-treatment differences for second-line therapy compared with 
first-line therapy. This implies a larger benefit for combination therapy over statins alone for patients already on statins (second-line therapy patients).

In summary, our analysis results with this rich dataset reveal the following: (i) statin therapy is effective, but statins in combination with EZE are even more effective in reducing LDL$\mathrm{C}$; (ii) the treatment difference (for \% change from baseline) was higher in the second-line studies and can be attributed, in part, to lower baseline LDL-C values; (iii) baseline LDL-C, baseline HDL-C, and study duration are important covariates in LDL-C reduction; (iv) while conducting an aggregate meta-analysis, it is critical to incorporate study-level covariates, which is not a common practice, and the number of covariates that can be included in the model is dependent upon the number of studies included in the meta-analysis; and (v) even though the study-level estimates used in the meta-analysis may be adjusted locally (within study) for some of the covariates, it is still critical to incorporate these covariates in the meta-analysis model; in other words, adjusting for covariates at the study level is not sufficient.

As the model fitting is now done at the study level, one must be careful not to fit too large of a meta-regression model relative to the number of studies, and the results should be interpreted with caution. In our case study, we had 26 studies, and each study had two treatment arms, which yielded 52 data points. The largest model we fit contained 18 parameters, and so we had 34 degrees of freedom left over. In the meta-analysis literature, there are no current guidelines discussing how large of a model to fit relative to the number of studies. One needs to use common sense and practical judgment and fit models that yield stable estimates and SEs, especially for the variance components. In our case study, the estimates from the largest model were quite stable, and the EM algorithm always converged in relatively few iterations, thus giving us confidence that the models we fit were reasonable. On the basis of our experience with these data, we would recommend a general rule of thumb for model fitting that $n \geq 2 q$, where $n$ denotes the number of data points and $q$ denotes the total number of parameters in the meta-regression model. In carrying out a variable selection procedure, there may be an inherent multiplicity issue in the covariate selection procedure, and as a result one must bear this in mind when carrying out such a procedure and perhaps conduct simulation studies to examine whether the type I error is controlled with a given procedure. At the very least, this issue must be acknowledged by the data analyst.

In Section 3.1, we assume that $w_{j k}$ is a known precision. Because we have only a single observation for the $j$ th treatment within the $k$ th trial, $w_{j k}$ is not estimable. We need additional information to estimate $w_{j k}$. Suppose that the standard deviation, $\mathrm{SD}_{j k}$, is available. Then, the method recently proposed by Yao et al. [30] can be used to relax the assumption that $w_{j k}$ is known. Specifically, (3.2) needs to be replaced by two equations:

$$
\varepsilon_{j k} \sim N\left(0, \frac{\sigma_{j k}^{2}}{n_{j k}}\right) \quad \text { (3.2a) }
$$

and

$$
\frac{\left(n_{j k}-1\right) \mathrm{SD}_{j k}^{2}}{\sigma_{j k}^{2}} \sim \chi_{n_{j k}-1}^{2}, \quad \text { (3.2b) }
$$

where $\sigma_{j k}^{2}$ is an unknown variance. As shown in [30], using the profile likelihood approach, the model using (3.1), (3.2a), and (3.2b) reduces to the model (3.1) when $\epsilon_{j k}$ follows a scaled 
Student- $t$ distribution with $n_{j k}-1$ degrees of freedom and scale parameter $w_{j k}^{-1}=\frac{\mathrm{SD}_{j k}^{2}}{n_{j k}}$ after profiling out $\sigma_{j k}^{2}$. When $n_{j k}$ is large, the Student- $t$ distribution is approximately normal.

Thus, the model using (3.1) and (3.2) with a fixed variance $w_{j k}^{-1}=\frac{\mathrm{SD}_{j k}^{2}}{n_{j k}}$ is approximately the same as the model defined by (3.1), (3.2a), and (3.2b) when $n_{j k}$ is relatively large. For the LDL-C data, the smallest sample size was 92 . Thus, we do not need to estimate $w_{j k}$, and

instead, we can simply set $w_{j k}^{-1}=\frac{\mathrm{SD}_{j k}^{2}}{n_{j k}}$.

We mention here that the proposed methodology is quite general and can be applied in any meta-analysis setting including a wide range of scientific applications. We have focused here specifically on cholesterol-lowering drugs because of our collaborations in this area and the availability of several datasets. Future work includes extending the proposed methodology to multivariate responses in meta-analysis models, such as joint modeling of the percent reduction of LDL-C from baseline, the percent change of HDL-C from baseline, and the percent reduction of TG from baseline, on the basis of individual patient data or study-level data. Covariate data are commonly missing at the study level in meta-analysis, such as body mass index. The literature for analyzing such missing data is quite sparse. Currently, these clinically important extensions are under investigation.

\section{Acknowledgments}

We wish to thank the editor, the associate editor, and the two anonymous reviewers for their helpful comments and suggestions, which have led to an improvement of this article. Dr. Chen and Dr. Ibrahim's research was partially supported by NIH grants \#GM 70335 and \#CA 74015.

\section{References}

1. Whitehead A, Whitehead J. A general parametric approach to the meta-analysis of randomized clinical trials. Statistics in Medicine. 1991; 10:1665-1677. [PubMed: 1792461]

2. Hardy RJ, Thompson SG. A likelihood approach to meta-analysis with random effects. Statistics in Medicine. 1996; 15:619-629. [PubMed: 8731004]

3. Higgns JPT, Whitehead A. Borrowing strength from external trials in a meta-analysis. Statistics in Medicine. 1996; 15:2733-2749. [PubMed: 8981683]

4. Biggerstaff BJ, Tweedie RL. Incorporating variability in estimates of heterogeneity in the random effects model in meta-analysis. Statistics in Medicine. 1997; 16:753-768. [PubMed: 9131763]

5. Aitkin M. Meta-analysis by random effect modeling in generalized linear models. Statistics in Medicine. 1999; 18:2343-2351. [PubMed: 10474144]

6. Normand SL. Tutorial in biostatistics meta-analysis: formulating, evaluating, combining, and reporting. Statistics in Medicine. 1999; 18:321-359. [PubMed: 10070677]

7. Brockwell SE, Gordon IR. A comparison of statistical methods for meta-analysis. Statistics in Medicine. 2001; 20:825-840. [PubMed: 11252006]

8. Lopes HF, Müller P, Rosner GL. Bayesian meta-analysis for longitudinal data models using multivariate mixture priors. Biometrics. 2003; 59:66-75. [PubMed: 12762442]

9. Burr D, Doss H. A Bayesian semiparametric model for random-effects meta-analysis. Journal of the American Statistical Association. 2005; 100:242-251.

10. Hoskins JM, Goldberg RM, Qu P, Ibrahim JG, McLeod HL. UGT1A1*28 and irinotecan-induced neutropenia: dose matters. Journal of the National Cancer Institute. 2007; 99:1290-1295. [PubMed: 17728214]

11. Sutton AJ, Higgins J. Recent developments in meta-analysis. Statistics in Medicine. 2008; 27:625650. [PubMed: 17590884] 
12. Higgins JPT, Whitehead A, Simmonds MC. Sequential methods for random-effects meta-analyses. Statistics in Medicine. 2011; 30:903-921. [PubMed: 21472757]

13. Silva MA, Swanson AC, Gandhi PJ, Tataronis GR. Statin-related adverse events: a meta-analysis. Clinical Therapeutics. 2006; 28:26-35. [PubMed: 16490577]

14. Avis HJ, Vissers MN, Stein EA, Wijburg FA, Trip MD, Kastelein JJP, Hutten BA. A systematic review and meta-analysis of statin therapy in children with familial hypercholesterolemia. Arteriosclerosis, Thrombosis, and Vascular Biology. 2007; 27:1803-1810.

15. Cholesterol Treatment Trailists' (CTT) Collaborators. Efficacy of cholesterol-lowering therapy in 18,686 people with diabetes in 14 randomized trials of statins. Lancet. 2008; 371:117-125. [PubMed: 18191683]

16. Brugts JJ, Yetgin T, Hoeks SE, Gotto AM, Shepherd J, Westendrop RGJ, de Craen AJM, Knopp RH, Nakamura H, Ridker P, van Domburg R, Deckers JW. The benefits of stains in people without established cardiovascular disease but with cardiovascular risk factors: meta-analysis of randomised controlled trials. British Medical Journal. 2009; 338:b2376. [PubMed: 19567909]

17. Mikhailidis DP, Sibbring GC, Ballantyne CM, Davies GM, Catapano AL. Meta-analysis of the cholesterol-lowering effect of ezetimibe added to ongoing statin therapy. Current Medical Research and Opinion. 2007; 23:2009-2026. [PubMed: 17659159]

18. Catapano A, Brady WE, King TR, Palmisano J. Lipid altering-efficacy of ezetimibe coadministered with simvastatin compared with rosuvastatin: a meta-analysis of pooled data from 14 clinical trials. Current Medical Research and Opinion. 2005; 21:1123-1130. [PubMed: 16004682]

19. Thompson SG, Higgins JPT. How should meta-regression analyses be undertaken and interpreted? Statistics in Medicine. 2002; 21:1559-1573. [PubMed: 12111920]

20. Knapp G, Hartung J. Improved tests for a random effects meta-regression with a single covariate. Statistics in Medicine. 2003; 22:2693-2710. [PubMed: 12939780]

21. Simmonds MC, Higgins J. Covariate heterogeneity in meta-analysis: criteria for deciding between meta-regression and individual patient data. Statistics in Medicine. 2007; 26:2982-2999. [PubMed: 17195960]

22. Harbord RM, Higgins J. Meta-regression in Stata. The Stata Journal. 2008; 8:493-519.

23. Jackson D. The significance level of meta-regression's standard hypothesis test. Communications in Statistics - Theory and Methods. 2008; 37:1576-1590.

24. Hedges LV, Tipton E, Johnson MC. Robust variance estimation in meta-regression with dependent effect size estimates. Research Synthesis Methods. 2010; 1:39-65.

25. Hemming K, Hutton JL, Maguire MG, Marson AG. Meta-regression with partial information on summary trial or patient characteristics. Statistics in Medicine. 2010; 29:1312-1324. [PubMed: 20087842]

26. White IR. Multivariate random-effects meta-regression: updates to mvmeta. The Stata Journal. 2011; 11:255-270.

27. Cochran WG. The combination of estimates from different experiments. Biometrics. 1954; 10:101-129.

28. Hurvich CM, Tsai CL. Regression and time series model selection in small samples. Biometrika. 1989; 76:297-307.

29. Burnham, KP.; Anderson, DR. Model Selection and Inference: A Practical Information-Theoretic Approach. Springer-Verlag; New York: 1998.

30. Yao H, Chen MH, Qiu C. Bayesian modeling and inference for meta data with applications in efficacy evaluation of an allergic rhinitis drug. Journal of Biopharmaceutical Statistics. 2011; 21:992-1005. [PubMed: 21830927]

31. Ibrahim JG. Incomplete data in generalized linear models. Journal of the American Statistical Association. 1990; 85:765-769.

32. Ibrahim JG, Chen MH, Lipsitz SR. Missing responses in generalized linear mixed models when the missing data mechanism is nonignorable. Biometrika. 2001; 88:551-564.

33. Louis T. Finding the observed information matrix when using the EM algorithm. Journal of the Royal Statistical Society, Series B. 1982; 44:226-233. 
34. Ibrahim JG, Lipsitz SR, Chen MH. Missing covariates in generalized linear models when the missing data mechanism is nonignorable. Journal of the Royal Statistical Society, Series B. 1999; 61:173-190.

35. Schott, JR. Matrix Analysis for Statistics. Wiley; New York: 1997.

\section{Appendix: The complete-data likelihood function and expectation- maximization algorithm for meta-regression models \\ The complete-data likelihood function}

Let $\xi=\left(\boldsymbol{\xi}_{1}^{\prime}, \boldsymbol{\xi}_{2}^{\prime}, \ldots, \boldsymbol{\xi}_{K}^{\prime}\right)^{\prime}$ be the vector of unknown latent variables as defined in (3.3). Then, the conditional distribution of $\boldsymbol{y}_{k}$ given $\varphi$ and $\boldsymbol{\xi}_{k}$ is of the form

$$
\begin{aligned}
f\left(\boldsymbol{y}_{k} \mid X_{k}, Z_{k}, \boldsymbol{\phi}, \xi_{k}\right) & \\
& =\prod_{j=1}^{J}\left(\frac{w_{j k}}{2 \pi}\right)^{1 / 2} \exp \left\{-\frac{w_{j k}}{2}\left(y_{j k}-\tilde{\boldsymbol{x}}_{j k}^{\prime} \boldsymbol{\phi}-\boldsymbol{z}_{j k}^{\prime} \boldsymbol{\xi}_{k}\right)^{2}\right\} \\
& =\left\{\prod_{j=1}^{J}\left(\frac{w_{j k}}{2 \pi}\right)^{1 / 2}\right\} \exp \left\{-\frac{1}{2}\left(\boldsymbol{y}_{k}-X_{k} \boldsymbol{\phi}-Z_{k} \boldsymbol{\xi}_{k}\right)^{\prime} W_{k}\left(\boldsymbol{y}_{k}-X_{k} \boldsymbol{\phi}-Z_{k} \boldsymbol{\xi}_{k}\right)\right\},
\end{aligned}
$$

where $X_{k}=\left(x_{\Psi_{k}}, x_{k}\right)^{\prime}$, and conditional on $V, \xi_{k}$ has the density

$f\left(\xi_{k} \mid V\right)=\frac{|V|^{-1 / 2}}{(2 \pi)^{J}} \exp \left(-\frac{1}{2} \xi_{k}^{\prime} V^{-1} \xi_{k}\right)$. Ignoring the constants that are free of $\boldsymbol{\eta}$ and $\exp \boldsymbol{\xi}_{k}$, the complete-data log-likelihood is given by

$$
\ell(\eta, \xi)=\sum_{k=1}^{K} \ell\left(\eta ; \boldsymbol{y}_{k}, \xi_{k}\right)=\sum_{k=1}^{K}\left[\left\{-\frac{1}{2}\left(\boldsymbol{y}_{k}-X_{k} \boldsymbol{\phi}-Z_{k} \boldsymbol{\xi}_{k}\right)^{\prime} W_{k}\left(\boldsymbol{y}_{k}-X_{k} \boldsymbol{\phi}-Z_{k} \boldsymbol{\xi}_{k}\right)\right\}+\left(-\frac{1}{2} \log |V|-\frac{1}{2} \boldsymbol{\xi}_{k}^{\prime} V^{-1} \boldsymbol{\xi}_{k}\right)\right] .
$$

\section{Expectation-maximization algorithm for meta-regression models}

We develop an efficient EM algorithm to compute the maximum likelihood estimates of $\boldsymbol{\eta}$ via the EM by the method of Weights, which was initially introduced by Ibrahim [31] and further developed by Ibrahim, Chen, and Lipsitz [32] in the context of generalized linear models with non-ignorable missing covariate data. The algorithm involves two steps: the Estep and the M-step. Let $\eta^{(t)}=\left(\varphi^{(t)},\left(V^{-1}\right)^{(t)}\right)$ denote the value of $\boldsymbol{\eta}$ at the $t$ th EM iteration. Note that at the initial EM iteration, $V^{(0)}$ must be chosen to be a block diagonal matrix with a structure similar to $V$. To compute the E-step, we must compute the expectation of the complete-data log-likelihood (A.1) with respect to the conditional distribution of the latent variables $\xi_{k}$ given the observed data. We can write the E-step for the observations from the $k$ th trial at the $(t+1)$ st EM iteration as

$$
\begin{aligned}
& Q_{k}\left(\eta \mid \eta^{(t)}\right)=\int \ell\left(\eta ; \boldsymbol{y}_{k}, \boldsymbol{\xi}_{k}\right) f\left(\boldsymbol{\xi}_{k} \mid \boldsymbol{y}_{k}, \eta^{(t)}\right) d \boldsymbol{\xi}_{k} \\
& \quad=\int\left\{-\frac{1}{2}\left(\boldsymbol{y}_{k}-X_{k} \boldsymbol{\phi}-Z_{k} \boldsymbol{\xi}_{k}\right)^{\prime} W_{k}\left(\boldsymbol{y}_{k}-X_{k} \boldsymbol{\phi}-Z_{k} \boldsymbol{\xi}_{k}\right)\right\} f\left(\boldsymbol{\xi}_{k} \mid \boldsymbol{y}_{k}, \eta^{(t)}\right) d \boldsymbol{\xi}_{k}+ \\
& \quad \int\left(-\frac{1}{2} \log |V|-\frac{1}{2} \boldsymbol{\xi}_{k}^{\prime} V^{-1} \boldsymbol{\xi}_{k}\right) f\left(\boldsymbol{\xi}_{k} \mid \boldsymbol{y}_{k}, \eta^{(t)}\right) d \boldsymbol{\xi}_{k},
\end{aligned}
$$


where $f\left(\boldsymbol{\xi}_{k} \mid \boldsymbol{y}_{k}, \eta^{(t)}\right)$ is the conditional distribution of $\boldsymbol{\xi}_{k}$ given the observed data $\boldsymbol{y}_{k}$ and the value of $\boldsymbol{\eta}$ at the $t$ th EM iteration. After much tedious algebra, it can be shown that $\xi_{k} \mid y_{k}$,

$\xi_{k} \mid \boldsymbol{y}_{k}, \eta^{(t)} \sim N\left(\xi_{k}^{(t)}, \sum_{k}^{(t)}\right)$, where $\xi_{k}^{(t)}=\sum_{k}^{(t)} Z_{k}^{\prime} W_{k}\left(\boldsymbol{y}_{k}-X_{k} \phi^{(t)}\right)$ and

$\sum_{k}^{(t)}=\left\{Z_{k}^{\prime} W_{k} Z_{k}+\left(V^{(t)}\right)^{-1}\right\}^{-1}$. After some algebra, $Q_{k}\left(\boldsymbol{\eta} \mid \eta^{(t)}\right)$ reduces to

$$
\begin{aligned}
Q_{k} & \left(\eta \mid \eta^{(t)}\right) \\
& =-\frac{1}{2}\left\{\left(y_{k}-X_{k} \boldsymbol{\phi}-Z_{k} \boldsymbol{\xi}_{k}^{(t)}\right)^{\prime} W_{k}\left(\boldsymbol{y}_{k}-X_{k} \boldsymbol{\phi}-Z_{k} \boldsymbol{\xi}_{k}^{(t)}\right)+\operatorname{tr}\left(Z_{k}^{\prime} W_{k} Z_{k} \sum_{k}^{(t)}\right)\right\} \\
& -\frac{1}{2} \log |V| \\
& -\frac{1}{2} \boldsymbol{\xi}_{k}^{(t)^{\prime}} V^{-1} \xi_{k}^{(t)} \\
& -\frac{1}{2} \operatorname{tr}\left(V^{-1} \sum_{k}^{(t)}\right) .
\end{aligned}
$$

Thus, the E-step for all the observations from the $K$ trials is given by $Q\left(\eta \mid \eta^{(t)}\right)=\sum_{k=1}^{K} Q_{k}\left(\eta \mid \eta^{(t)}\right)$.

The M-step maximizes $Q\left(\eta \eta^{(t)}\right)$, and closed forms for the parameter estimates are available. Specifically, the M-step is obtained as follows. First, we find $V^{(t+1)}$ to maximize

$$
Q_{V}=-\frac{K}{2} \log |V|-\frac{1}{2} \sum_{k=1}^{K} \boldsymbol{\xi}_{k}^{(t)^{\prime}} V^{-1} \boldsymbol{\xi}_{k}^{(t)}-\frac{1}{2} \sum_{k=1}^{K} t r\left(V^{-1} \sum_{k}^{(t)}\right)
$$

which gives

$$
V^{(t+1)}=\frac{1}{K} \sum_{k=1}^{K}\left\{\boldsymbol{\xi}_{k}^{(t)} \boldsymbol{\xi}_{k}^{(t)^{\prime}}+\sum_{k}^{(t)}\right\}
$$

Second, we find $\varphi^{(t+1)}$ to minimize $Q_{\boldsymbol{\phi}}=\sum_{k=1}^{K}\left(\boldsymbol{y}_{k}-X_{k} \phi-Z_{k} \boldsymbol{\xi}_{k}^{(t)}\right)^{\prime} W_{k}\left(\boldsymbol{y}_{k}-X_{k} \phi-Z_{k} \boldsymbol{\xi}_{k}^{(t)}\right)$, which yields

$$
\phi^{(t+1)}=\left(\sum_{k=1}^{K} X_{k}^{\prime} W_{k} X_{k}\right)^{-1} \sum_{k=1}^{K} X_{k}^{\prime} W_{k}\left(\boldsymbol{y}_{k}-Z_{k} \boldsymbol{\xi}_{k}^{(t)}\right)
$$

Let $\boldsymbol{\eta}$ /denote the estimate of $\eta$ at EM convergence. To obtain the asymptotic covariance matrix of $\eta$, we use the method of Louis [33]. Following a similar derivation as in [34], the estimated observed information matrix of $\eta$ is given by 


$$
\mathscr{I}(\widehat{\eta})=-\ddot{Q}(\widehat{\eta} \mid \widehat{\eta})-\left\{\sum_{k=1}^{K} E\left[S_{k}\left(\widehat{\eta} ; \boldsymbol{y}_{k}, \boldsymbol{\xi}_{k}\right) S_{k}\left(\widehat{\eta} ; \boldsymbol{y}_{k}, \boldsymbol{\xi}_{k}\right)^{\prime} \mid \boldsymbol{y}_{k}, \widehat{\eta}\right]=\sum_{k=1}^{N} \dot{Q}_{k}(\widehat{\eta} \mid \widehat{\eta}) \dot{Q}_{k}(\widehat{\eta} \mid \widehat{\eta})^{\prime}\right\}, \quad \begin{array}{r}
\text { (A. } \\
2)
\end{array}
$$

where $\eta$ is the estimate of $\eta$ at convergence,

$$
S_{k}\left(\widehat{\eta} ; \boldsymbol{y}_{k}, \xi_{k}\right)=\left[\frac{\partial \ell\left(\eta: \boldsymbol{y}_{k}, \boldsymbol{\xi}_{k}\right)}{\partial \eta}\right]_{\eta=\hat{\eta}}
$$

$Q$ denotes the first derivative vector of $Q$ with respect to $\eta$, and $Q$ denotes the second derivative matrix of $Q$ with respect to $\eta$. The expectation in (A.2) is taken with respect to the density $f\left(\boldsymbol{\xi}_{k} \mid \boldsymbol{y}_{k}, \eta\right)$.

The estimate of the asymptotic covariance matrix of $\varphi^{\text {is }}$ the upper $p^{*} \times p^{*}$ block of [ $\mathcal{I}$ $\left.(\eta)^{-1}\right]^{-1}$, where $p^{*}=p+2 J$ is the dimension of $\varphi$. Straightforward derivations yield

$$
\frac{\partial Q_{k}\left(\eta \mid \eta^{(t)}\right)}{\partial \phi}=X_{k}^{\prime} W_{k}\left(\boldsymbol{y}_{k}-Z_{k} \boldsymbol{\xi}_{k}^{(t)}\right)-\left(X_{k}^{\prime} W_{k} X_{k}\right) \phi \text { and } \frac{\partial^{2} Q_{k}\left(\eta \mid \eta^{(t)}\right)}{\partial \phi} \partial \phi^{\prime}=-X_{k}^{\prime} W_{k} X_{k} .
$$

Write $V^{*}=V^{-1}, V^{*}=\operatorname{diag}\left(V_{1}^{*}, V_{2}^{*}\right)$, where $V_{\ell}^{*}=V_{\ell}^{-1}=\left(V_{\ell j j^{\prime}}^{*}\right)_{J \times J}$ for $\ell=1$, 2. Let

$$
v\left(V_{\ell}^{*}\right)=\left(V_{\ell 11}^{*}, V_{\ell 21}^{*}, \ldots, V_{\ell J 1}^{*}, V_{\ell 22}^{*}, \ldots, V_{\ell J 2}^{*}, \ldots, V_{\ell J J}^{*}\right)^{\prime}
$$

which is formed by stacking the columns of the lower triangular portion of $V_{\ell}^{*}$ and

$$
\operatorname{vec}\left(V_{\ell}^{*}\right)=\left(V_{\ell 11}^{*}, \ldots, V_{\ell J 1}^{*}, \ldots, V_{\ell 12}^{*}, \ldots, V_{\ell J 2}^{*}, \ldots, V_{\ell 1 J}^{*}, \ldots, V_{\ell J J}^{*}\right)^{\prime}
$$

which is formed by stacking the columns of $V_{\ell}^{*}$. Then, we have

$$
\frac{\partial^{2} Q_{k}\left(\eta \mid \eta^{(t)}\right)}{\partial \gamma \partial v\left(V_{\ell}^{*}\right)^{\prime}}=\mathbf{0}
$$

for $\ell=1,2$. Notice that $\Sigma_{k}(t)$ is a block diagonal matrix. Then, we can write

$$
\begin{aligned}
Q_{k, V^{*}} & \left(\eta \mid \eta^{(t)}\right)=\frac{1}{2} \log \left|V^{*}\right|-\frac{1}{2} \boldsymbol{\xi}_{k}^{(t)} V^{\prime} V_{k}^{(t)}-\frac{1}{2} \operatorname{tr}\left(V^{*} \sum_{k}^{(t)}\right) \\
= & \frac{1}{2} \log \left|V_{1}^{*}\right|-\frac{1}{2} \boldsymbol{\xi}_{1 k}^{(t)^{\prime}} V_{1}^{*} \xi_{1 k}^{(t)}-\frac{1}{2} \operatorname{tr}\left(V_{1}^{*} \sum_{1 k}^{(t)}\right) \\
& +\frac{1}{2} \log \left|V_{2}^{*}\right|-\frac{1}{2} \boldsymbol{\xi}_{2 k}^{(t)^{\prime}} \boldsymbol{V}_{2}^{*} \xi_{2 k}^{(t)}-\frac{1}{2} \operatorname{tr}\left(V_{2}^{*} \sum_{2 k}^{(t)}\right),
\end{aligned}
$$


where $\xi_{k}^{(t)}=\left(\boldsymbol{\xi}_{1 k}^{(t)^{\prime}}, \boldsymbol{\xi}_{2 k}^{(t)^{\prime}}\right)^{\prime}, \sum_{k}^{(t)}=\operatorname{diag}\left(\sum_{1 k}^{(t)}, \sum_{2 k}^{(t)}\right)$, and $\boldsymbol{\xi}_{\ell k}^{(t)^{\prime}}$ is a $J$-dimensional vector, and $\sum_{\ell k}^{(t)}$ is a $J \times J$ symmetric matrix for $\ell=1,2$. Using the matrix derivative formula given in [35], we obtain

$$
\frac{\partial Q_{k}\left(\eta \mid \eta^{(t)}\right)}{\partial v\left(V_{\ell}^{*}\right)}=\frac{1}{2} D_{J}^{\prime}\left\{\operatorname{vec}\left(V_{\ell}^{*-1}\right)-\operatorname{vec}\left(\boldsymbol{\xi}_{\ell k}^{(t)} \boldsymbol{\xi}_{\ell k}^{(t)^{\prime}}+\sum_{\ell k}^{(t)}\right)\right\}
$$

where $D_{J}$ is the $\mathcal{J}^{2} \times J(J+1) / 2$ duplicated matrix such that $D_{J} v\left(V_{\ell}^{*}\right)=\operatorname{vec}\left(V_{\ell}^{*}\right)$. When $J=2$,

$$
D_{2}=\left(\begin{array}{lll}
1 & 0 & 0 \\
0 & 1 & 0 \\
0 & 1 & 0 \\
0 & 0 & 1
\end{array}\right)
$$

Again, using the matrix derivative formula given in [35], we have

$$
\frac{\partial^{2} Q_{k}\left(\eta \mid \eta^{(t)}\right)}{\partial v\left(V_{\ell}^{*}\right) \partial v\left(V_{\ell}^{*}\right)^{\prime}}=-\frac{1}{2} D_{J}^{\prime}\left(V_{\ell}^{*-1} \otimes V_{\ell}^{*-1}\right) D_{J}
$$

where $\square$ denotes the Kronecker product, for $\ell=1$, 2. Furthermore, we have $\frac{\partial^{2} Q_{K}\left(\eta \mid \eta^{(t)}\right)}{\partial v\left(V_{1}^{*}\right) \partial v\left(V_{2}^{*}\right)^{\prime}}=\mathbf{0}$. Using (A.3), (A.5), and (A.6) with both $\eta^{(t)}$ and $\eta$ replaced by $\eta^{\eta}$,we can easily obtain closed-form expressions of $Q(\eta n \mid \eta \eta)$ and $Q_{k}(\eta)(n)$.

Using the complete-data log-likelihood $\ell(\eta, \xi)$, we have

$$
\begin{aligned}
& \ell\left(\eta ; \boldsymbol{y}_{k}, \xi_{k}\right)=-\frac{1}{2}\left(y_{k}-X_{k} \boldsymbol{\phi}-Z_{k} \boldsymbol{\xi}_{k}\right)^{\prime} W_{k}\left(y_{k}-X_{k} \boldsymbol{\phi}-Z_{k} \xi_{k}\right)+\frac{1}{2} \log \left|V_{1}^{*}\right| \\
& -\frac{1}{2} \xi_{1 k}^{\prime} V_{1}^{*} \xi_{1 k}+\frac{1}{2} \log \left|V_{2}^{*}\right|-\frac{1}{2} \xi_{2 k}^{\prime} V_{2}^{*} \xi_{2 k},
\end{aligned}
$$

where $\xi_{\ell k}=\left(\xi_{\ell 1 k}, \ldots, \xi_{\ell J k}\right)^{\prime}$ for $\ell=1,2$. Similar to (A.3) and (A.5), we obtain

$$
S_{k, 1}=\frac{\partial \ell\left(\eta ; \boldsymbol{y}_{k}, \boldsymbol{\xi}_{k}\right)}{\partial \phi}=X_{k}^{\prime} W_{k}\left(\boldsymbol{y}_{k}-Z_{k} \xi_{k}\right)-\left(X_{k}^{\prime} W_{k} X_{k}\right) \phi
$$

and

$$
S_{k, 1+\ell}=\frac{\partial \ell\left(\eta ; \boldsymbol{y}_{k}, \boldsymbol{\xi}_{k}\right)}{\partial v\left(V_{\ell}^{*}\right)}=\frac{1}{2} D_{J}^{\prime}\left\{\operatorname{vec}\left(V_{\ell}^{*-1}\right)-\operatorname{vec}\left(\boldsymbol{\xi}_{\ell k} \boldsymbol{\xi}_{\ell k}^{\prime}\right)\right\}
$$


for $\boldsymbol{\ell}=1,2$. Write $Z_{k}=\left(Z_{1 k}, Z_{2 k}\right)$, where $Z_{1 k}=\left(\left(1-I_{k}\right) \boldsymbol{e}_{1}, \ldots,\left(1-I_{k}\right) \boldsymbol{e}_{J}\right)^{\prime}$ and $Z_{2 k}=\left(I_{k} \boldsymbol{e}_{1}\right.$, $\left.\ldots, I_{k} \boldsymbol{e}_{J}\right)^{\prime}$. After some tedious algebra, we obtain

$E\left[S_{k, 1} S_{k, 1}^{\prime} \mid \boldsymbol{y}_{k}, \widehat{\eta}^{(t)}\right]=X_{k}^{\prime} W_{k}\left\{\left(\boldsymbol{y}_{k}-X_{k} \boldsymbol{\phi}-Z_{k} \widehat{\boldsymbol{\xi}}_{k}^{(t)}\right)\left(\boldsymbol{y}_{k}-X_{k} \boldsymbol{\phi}-Z_{k} \widehat{\boldsymbol{\xi}}_{k}^{(t)}\right)^{\prime}+Z_{k} \sum_{k}^{(t)} Z_{k}^{\prime}\right\} W_{k} X_{k} \quad$ (A.

and

$$
\begin{gathered}
E\left[S_{k, 1} S_{k, 1+\ell}^{\prime} \mid \boldsymbol{y}_{k}, \widehat{\eta}^{(t)}\right]=\frac{1}{2}\left\{X_{k}^{\prime} W_{k}\left(\boldsymbol{y}_{k}-X_{k} \boldsymbol{\phi}-Z_{k} \widehat{\boldsymbol{\xi}}_{k}^{(t)}\right) \operatorname{vec}\left(V_{\ell}^{*-1}-\left(\boldsymbol{\xi}_{\ell k}^{(t)} \boldsymbol{\xi}_{\ell k}^{(t)^{\prime}}+\sum_{\ell k}^{(t)}\right)\right)^{\prime}\right. \\
\left.+X_{k}^{\prime} W_{k} Z_{\ell k}\left(\boldsymbol{\xi}_{\ell k}^{(t)^{\prime}} \otimes \sum_{\ell k}^{(t)}+\sum_{\ell k}^{(t)} \otimes \boldsymbol{\xi}_{\ell k}^{(t)^{\prime}}\right)\right\} D_{J}
\end{gathered}
$$

for $\ell=1,2$. Now, we let $K_{J J}$ denote a $\vec{J}^{2} \times J^{2}$ commutation matrix such that $\operatorname{vec}(A)=K_{J J}$ $\operatorname{vec}\left(A^{\prime}\right)$, where $A$ is a $J \times J$ matrix. Also let $N_{J}=\frac{1}{2}\left(I_{J^{2}}+K_{J J}\right)$, where $I_{J^{2}}$ is the $\mathcal{J}^{2} \times \mathcal{J}^{2}$ identity matrix. Note that

$$
\begin{aligned}
& \operatorname{vec}\left(\xi_{\ell k} \xi_{\ell k}^{\prime}\right)=\operatorname{vec}\left(\left(\xi_{\ell k}-\widehat{\xi}_{\ell k}^{(t)}\right)\left(\xi_{\ell k}-\widehat{\xi}_{\ell k}^{(t)}\right)^{\prime}\right)+\operatorname{vec}\left(\left(\xi_{\ell k}-\widehat{\xi}_{\ell k}^{(t)}\right) \widehat{\boldsymbol{\xi}}_{\ell k}^{(t) \prime}\right) \\
& \quad+\operatorname{vec}\left(\widehat{\xi}_{k}^{(t)}\left(\xi_{\ell k}-\widehat{\boldsymbol{\xi}}_{\ell k}^{(t)}\right)^{\prime}\right)+\operatorname{vec}\left(\widehat{\xi}_{\ell k}^{(t)} \widehat{\boldsymbol{\xi}}_{\ell k}^{(t) \prime}\right) \\
& \quad=\left(\xi_{\ell k}-\widehat{\xi}_{\ell k}^{(t)}\right) \otimes\left(\xi_{\ell k}-\widehat{\xi}_{\ell k}^{(t)}\right)+\widehat{\xi}_{\ell k}^{(t)} \otimes\left(\xi_{\ell k}-\widehat{\xi}_{\ell k}^{(t)}\right)+\left(\xi_{\ell k}-\widehat{\xi}_{\ell k}^{(t)}\right) \otimes \widehat{\xi}_{\ell k}^{(t)}+\widehat{\xi}_{\ell k}^{(t)} \otimes \widehat{\xi}_{\ell k}^{(t)}
\end{aligned}
$$

for $\ell=1,2$. Using Theorem 9.20 of [35] and after some algebra, we obtain

$$
\begin{aligned}
E[ & \left.S_{k, 1+\ell} S_{k, 1+\ell}^{\prime} \mid \boldsymbol{y}_{k}, \widehat{\eta}^{(t)}\right] \\
& =\frac{1}{4} D_{J}^{\prime}\left[\operatorname{vec}\left(V_{\ell}^{*-1}-\left(\boldsymbol{\xi}_{\ell k}^{(t)} \boldsymbol{\xi}_{\ell k}^{(t)^{\prime}}+\sum_{\ell k}^{(t)}\right)\right) \operatorname{vec}\left(V_{\ell}^{*-1}-\left(\boldsymbol{\xi}_{\ell k}^{(t)} \boldsymbol{\xi}_{\ell k}^{(t)^{\prime}}+\sum_{\ell k}^{(t)}\right)\right)^{\prime}\right. \\
& \left.+2 N_{J}\left\{\sum_{\ell k}^{(t)} \otimes \sum_{\ell k}^{(t)}+\sum_{\ell k}^{(t)} \otimes\left(\boldsymbol{\xi}_{\ell k}^{(t)} \boldsymbol{\xi}_{\ell k}^{(t)^{\prime}}\right)+\left(\boldsymbol{\xi}_{\ell k}^{(t)} \boldsymbol{\xi}_{\ell k}^{(t)^{\prime}}\right) \otimes \sum_{\ell k}^{(t)}\right\}\right] D_{J}
\end{aligned}
$$

for $\ell=1$, 2. Finally, we obtain

$E\left[S_{k, 2} S_{k, 3}^{\prime} \mid \boldsymbol{y}_{k}, \widehat{\eta}^{(t)}\right]=\frac{1}{4} D_{J}^{\prime} \operatorname{vec}\left(V_{1}^{*-1}-\left(\boldsymbol{\xi}_{1 k}^{(t)} \boldsymbol{\xi}_{1 k}^{(t)^{\prime}}+\sum_{1 k}^{(t)}\right)\right) \operatorname{vec}\left(V_{2}^{*-1}-\left(\boldsymbol{\xi}_{2 k}^{(t)} \boldsymbol{\xi}_{2 k}^{(t)^{\prime}}+\sum_{2 k}^{(t)}\right)\right)^{\prime} D_{J}$

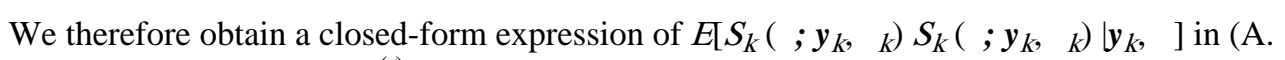
2) via (A.7)-(A.10) with $\eta^{(t)}$ and $\eta$ replaced by $\eta$ ? 


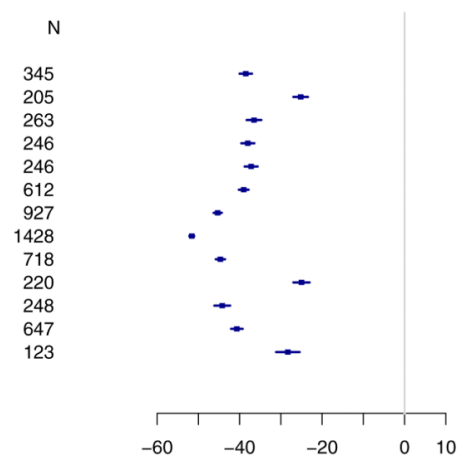

(a) First LineTherapy and Statin

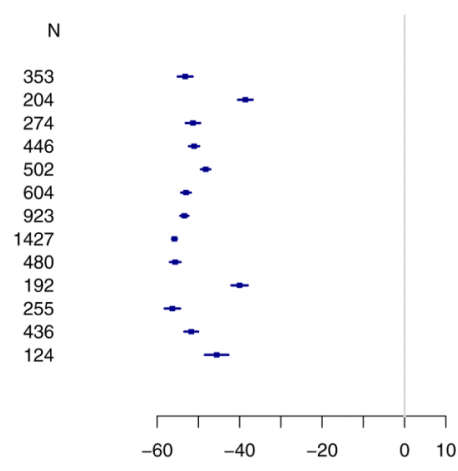

(b) First Line Therapy and Statin + EZE

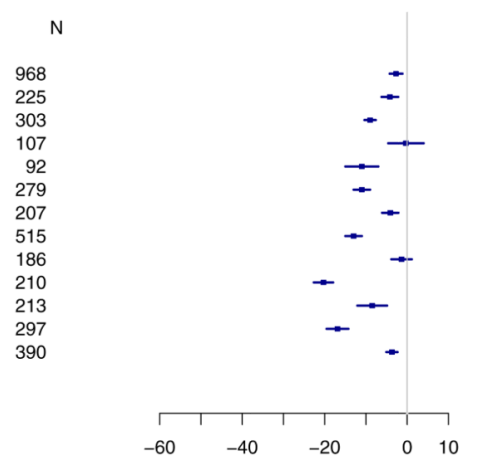

(c) Second LineTherapy and Statin

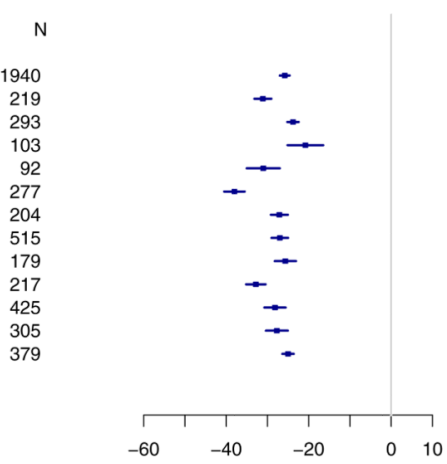

(d) Second Line Therapy and Statin + EZE

Figure 1.

Forest plots of LDL-C data, where each line corresponds to percent reduction in LDL-C from baseline with a $95 \%$ confidence interval. 


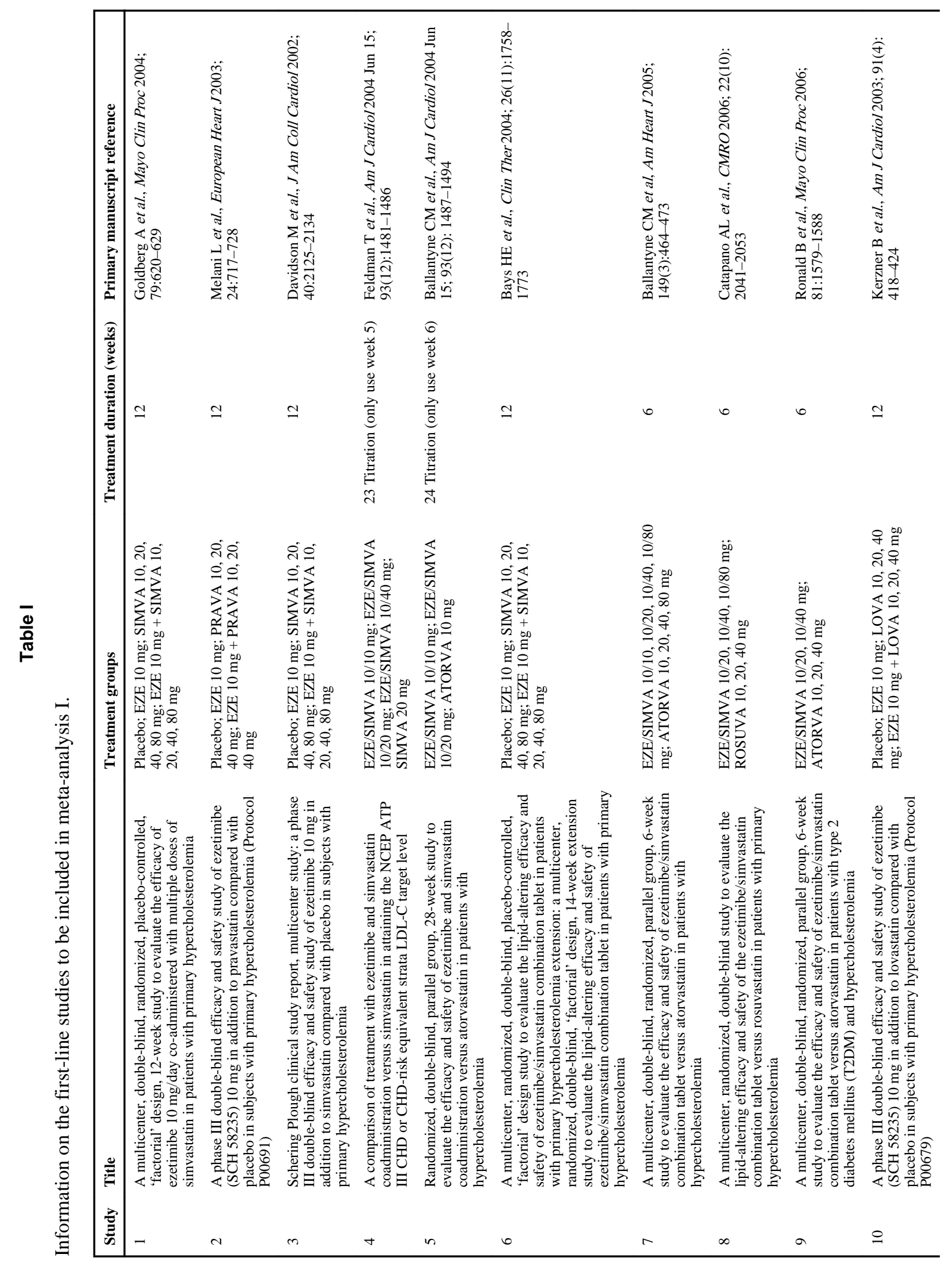

Stat Med. Author manuscript; available in PMC 2013 December 10. 


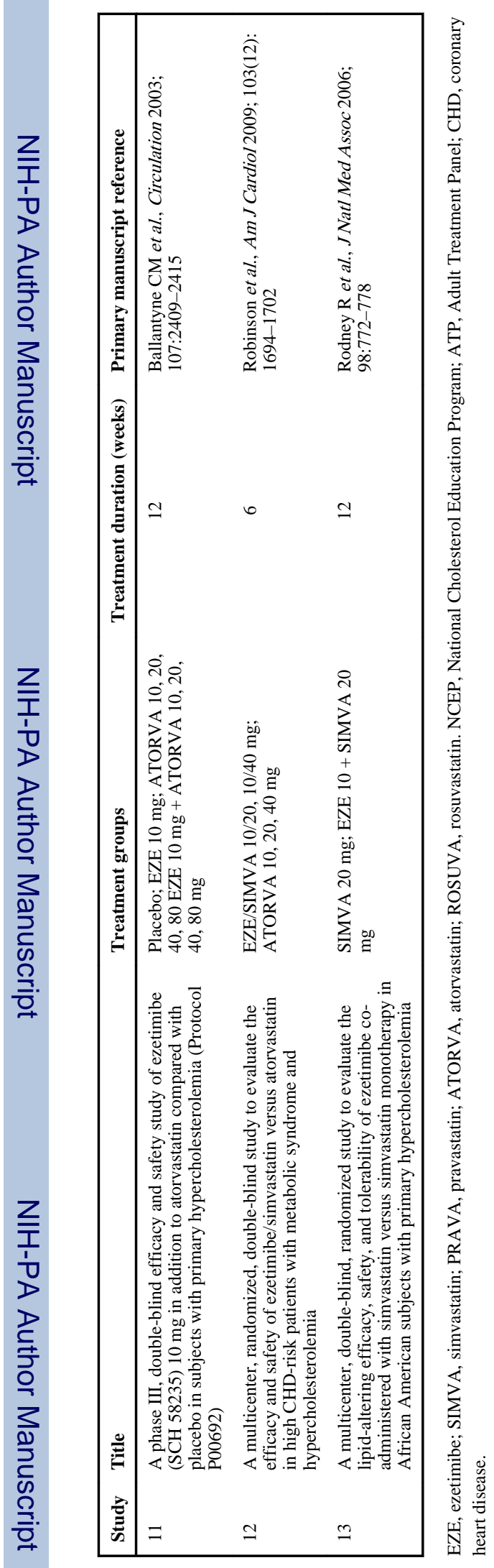

Stat Med. Author manuscript; available in PMC 2013 December 10. 


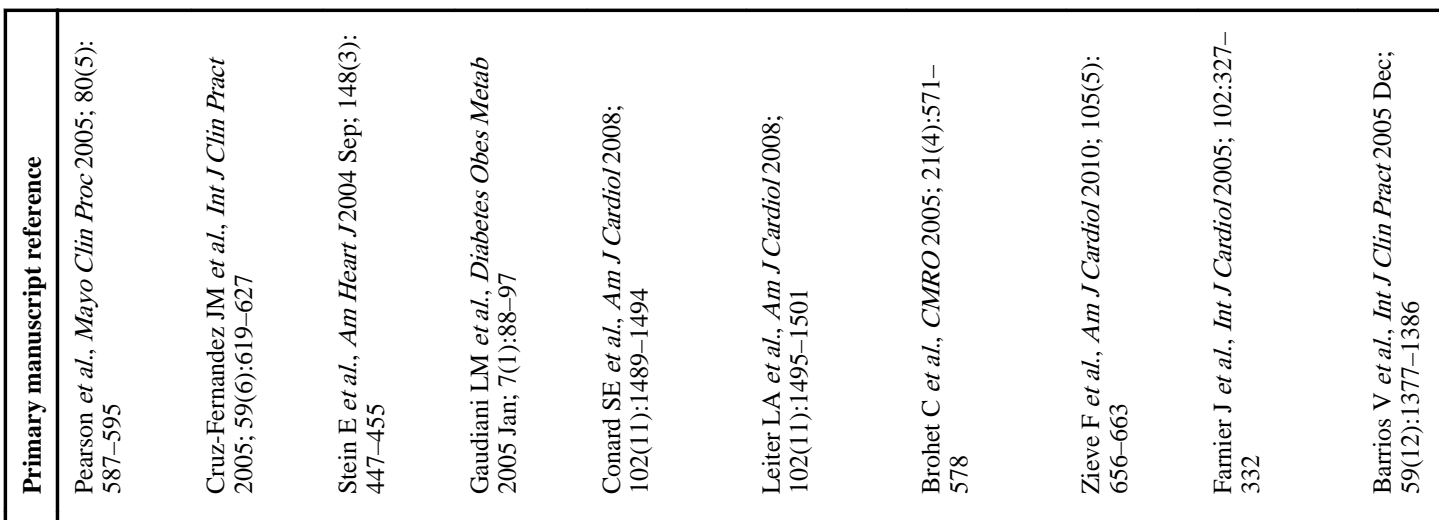

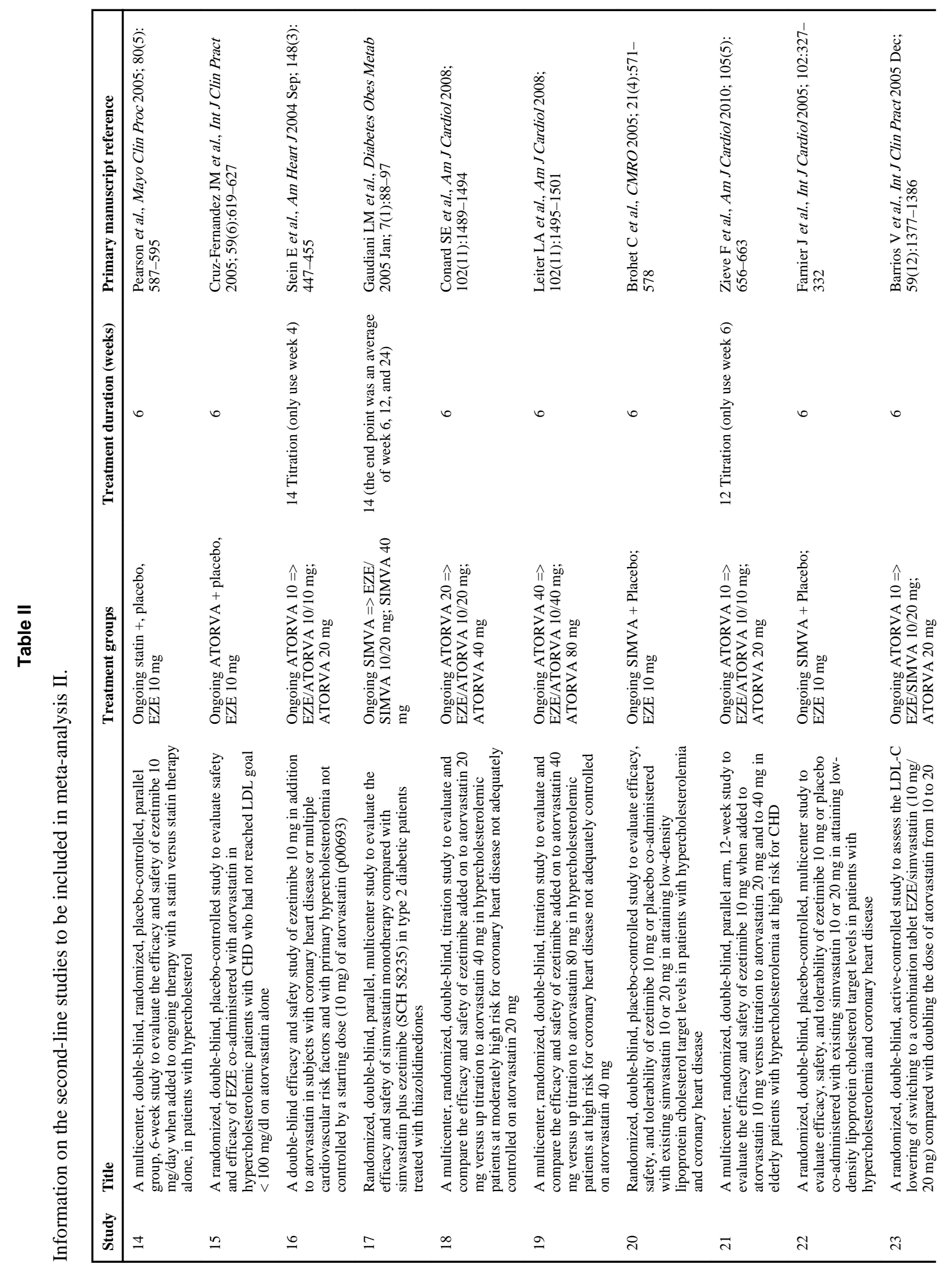

Stat Med. Author manuscript; available in PMC 2013 December 10. 


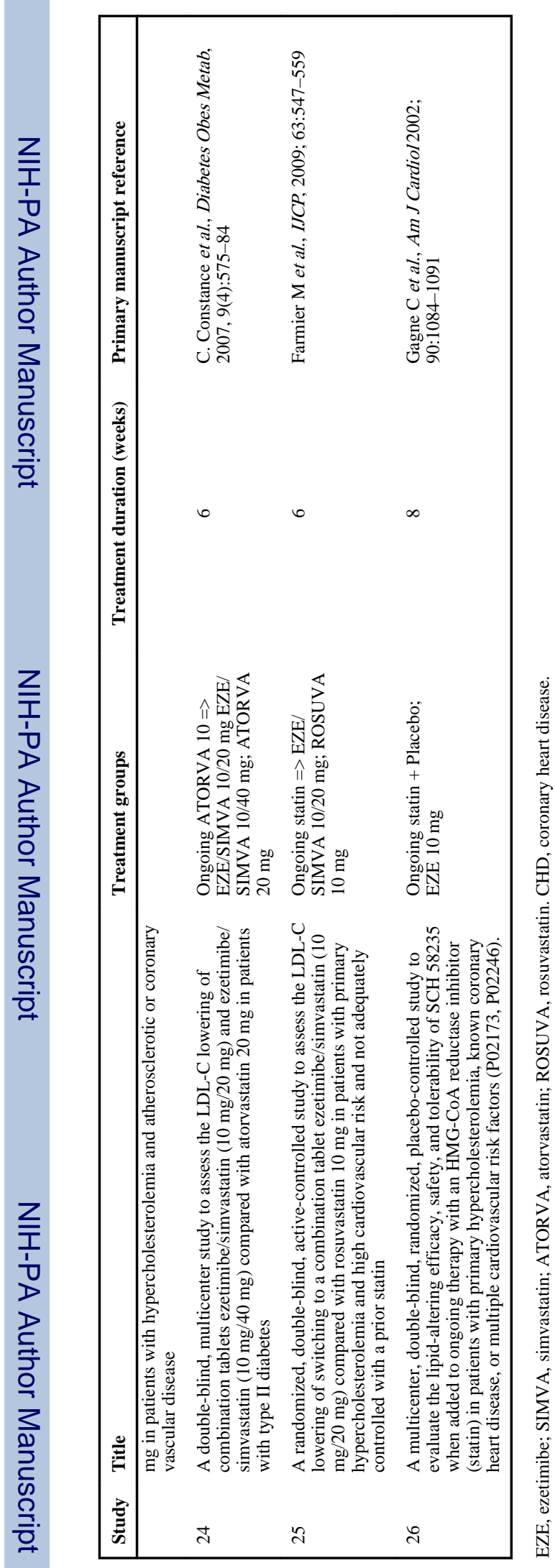

Stat Med. Author manuscript; available in PMC 2013 December 10. 


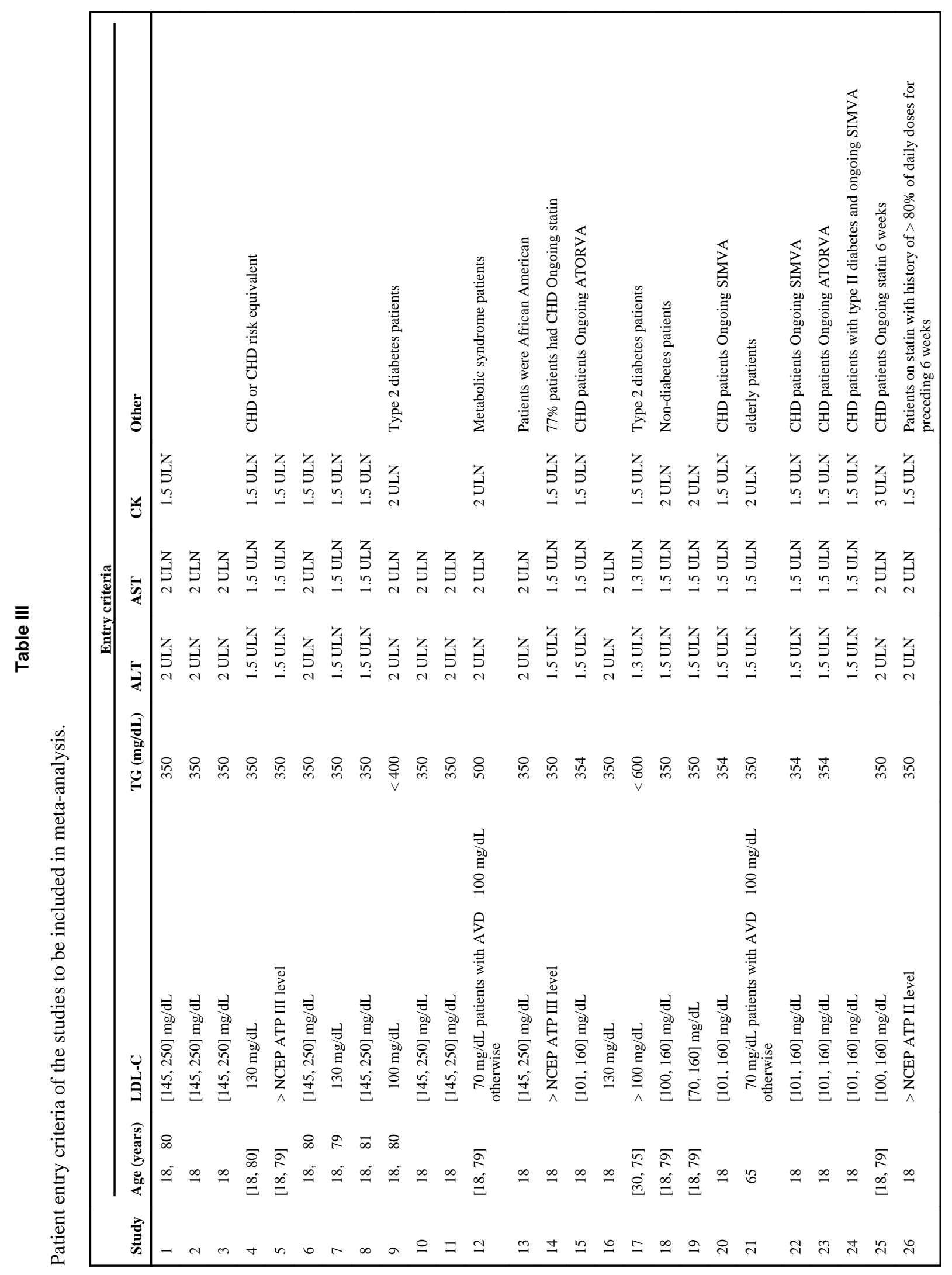




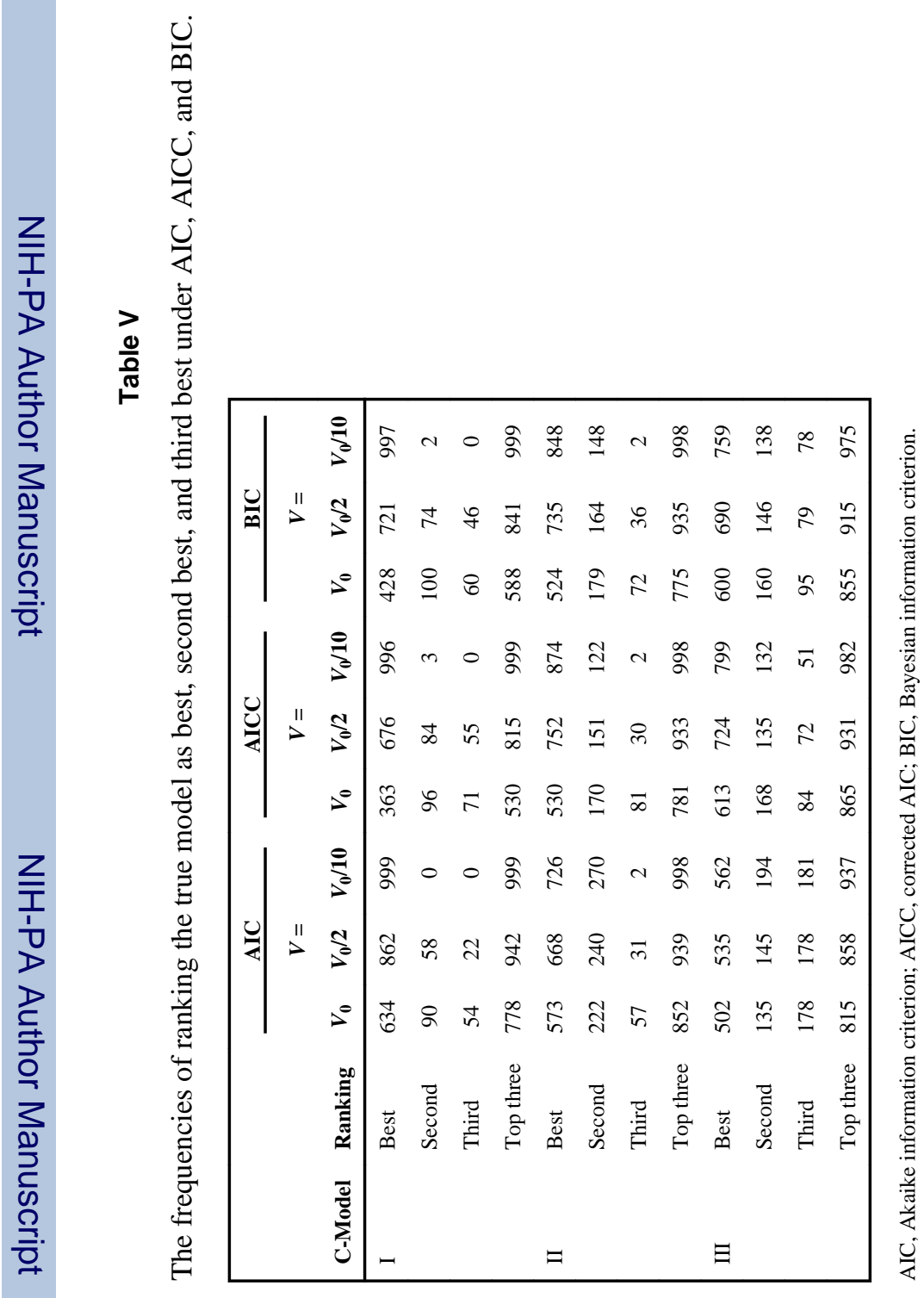

Stat Med. Author manuscript; available in PMC 2013 December 10. 
Table VI

The values of AIC, AICC, BIC, and $Q_{K}$ for the LDL-C data.

\begin{tabular}{|lcccc|}
\hline Model & AIC & AICC & BIC & $\boldsymbol{Q}_{\boldsymbol{K}}$ \\
\hline Baseline LDL-C, baseline HDL-C, duration & 315.7 & 325.3 & 332.1 & 1545.8 \\
Baseline LDL-C, duration & 316.6 & 324.6 & 331.7 & 2194.1 \\
Baseline LDL-C, baseline HDL-C, age, duration & 316.6 & 328.0 & 334.3 & 1362.1 \\
No covariates & 318.8 & 324.1 & 331.3 & 3033.6 \\
\hline
\end{tabular}

Note that the $p$-values associated with $Q K$ statistics were all $<0.0001$. 


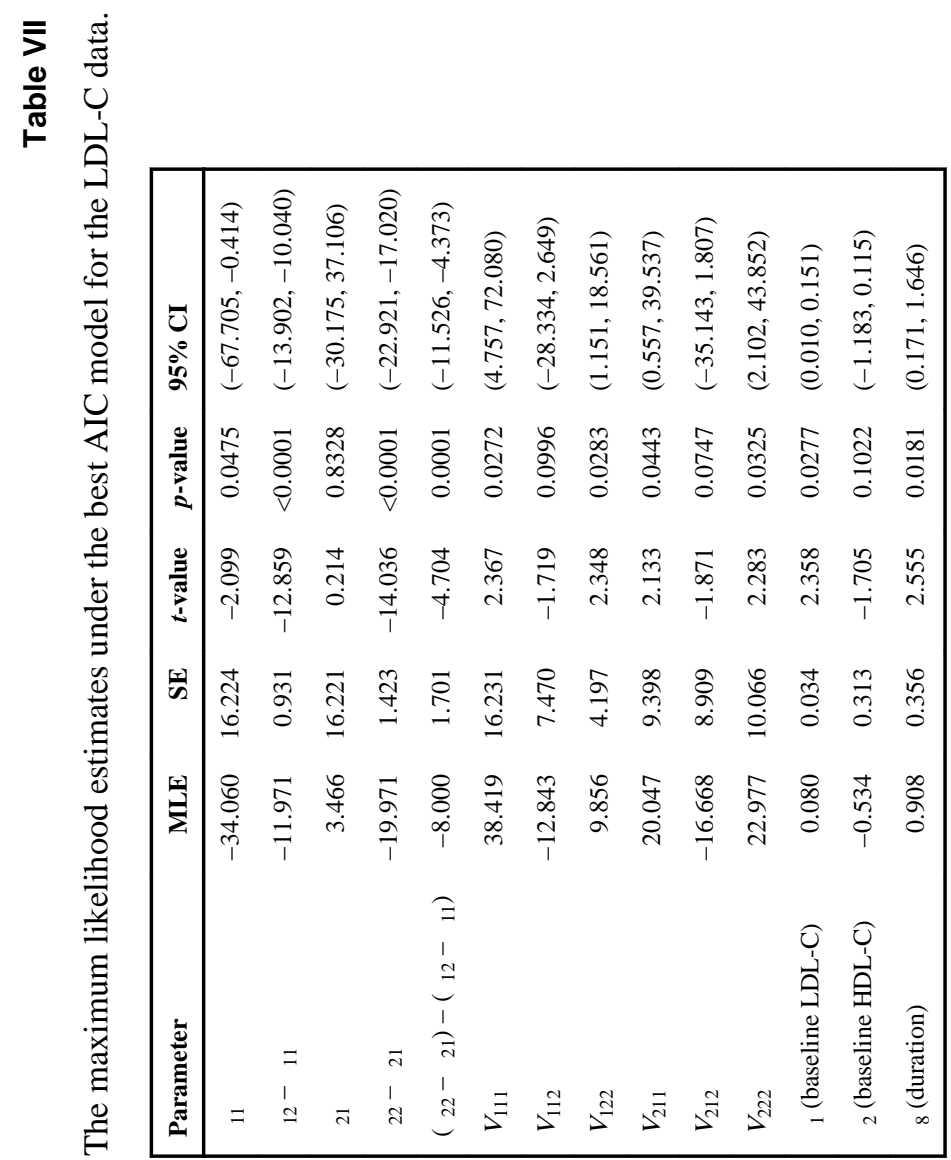

Stat Med. Author manuscript; available in PMC 2013 December 10. 